\title{
Fas-Associated Protein with Death Domain Regulates Notch Signaling during Muscle Regeneration
}

\author{
Rong Zhang ${ }^{a} \quad$ Lu Wang $^{a}$ Liangqiang He ${ }^{a}$ Bingya Yang ${ }^{a}$ Chun Yao ${ }^{a}$ Pan Du \\ Qiang $\mathrm{Xu}^{\mathrm{a}}$ Wei Cheng ${ }^{\mathrm{a}}$ Zi-Chun Hua ${ }^{\mathrm{a}, \mathrm{b}}$ \\ a State Key Laboratory of Pharmaceutical Biotechnology, College of Life Sciences and School of Stomatology, \\ Affiliated Stomatological Hospital, Nanjing University, Nanjing, and ${ }^{b}$ Changzhou High-Tech Research Institute of \\ Nanjing University and Jiangsu TargetPharma Laboratories Inc., Changzhou, PR China
}

\section{Key Words}

Fas-associated death domain $\cdot$ Mouse $\cdot$ Muscle

regeneration $\cdot$ Notch $\cdot$ Protein kinase $C \cdot$ Wnt

\begin{abstract}
Notch signaling plays critical roles during myogenesis by promoting the proliferation and inhibiting the differentiation of myogenic progenitors. However, the mechanism of the temporal regulation of Notch signaling during the myogenic lineage progression remains elusive. In the present study, we show that a constitutively phosphoryl-mimicking mutation of Fas-associated death domain (FADD-D) enhances Notch-1 signaling and compromises Wnt signaling in both cultured myoblasts and regenerating muscles, which results in inhibited myogenic differentiation and muscle regeneration. Inhibition of Notch signaling recovers the regeneration ability in injured FADD-D muscles through rescuing Wnt signaling. Furthermore, we found that protein kinase $\mathrm{Ca}$ mediates FADD-D-induced Notch-1 signaling by stabilizing Notch-1. Collectively, these data identify a novel mechanism for the temporal regulation of Notch signaling during myogenic lineage progression and muscle regeneration.
\end{abstract}

(c) 2015 S. Karger AG, Basel

\section{Introduction}

Notch signaling is activated immediately in the early phase of muscle regeneration. Studies have shown that the inhibition of Notch signaling impairs the proliferative expansion and myogenic progression of myoblasts [Conboy et al., 2003]. In addition to Notch, previous studies have indicated that fibroblast growth factors also promote myoblast activation and proliferation, which in turn promotes muscle regeneration [Anderson et al., 1995; Husmann et al., 1996; Chakkalakal et al., 2012; Yin et al.,

\section{Abbreviations used in this paper}

CHX cycloheximide

cPKC conventional protein kinase $\mathrm{C}$

FADD Fas-associated death domain

FADD-D constitutively phosphoryl-mimicking mutation of

$$
\text { FADD }
$$

NICD intracellular domain of Notch-1

PKC protein kinase C

TA tibialis anterior

WT wild-type

\section{KARGER}

E-Mail karger@karger.com

www.karger.com/cto
(C) 2015 S. Karger AG, Basel

1422-6405/15/2004-0253\$39.50/0
Zi-Chun Hua or Wei Cheng

Hankou Road 22

Nanjing University

Nanjing, Jiangsu Province 210093 (PR China)

E-Mail zchua@ nju.edu.cn or chengwe@ outlook.com 
2013]. Given that Notch signaling represses the differentiation of myoblasts, it should be compromised at a certain time to assure myogenic progression. A signaling switch from Notch to Wnt has been revealed as critical for myogenic lineage progression [Brack et al., 2008]. Wnt signaling is activated in the later phases of myogenic lineage progression to facilitate differentiation events [Otto et al., 2008]. However, the regulatory mechanisms involved in this switch have yet to be elucidated.

Protein kinase $\mathrm{C}$ (PKC) controls diverse biological processes, such as proliferation and differentiation, and is activated by secondary messengers generated by growth factors [Newton, 2010]. PKC conveys extracellular signals to initiate a cascade of cellular events, and evidence from diverse cell types has demonstrated that PKC regulates cell cycle progression to control proliferation and differentiation [Black, 2000]. We have shown that Fasassociated death domain (FADD) phosphorylation regulates conventional PKC ( $\mathrm{CPKC})$ signaling termination and cell migration [Cheng et al., 2012]. Moreover, we have also established the role of FADD phosphorylation in muscle stem cell self-renewal and commitment, which occurs mainly through cPKC-mediated Notch signaling [Cheng et al., 2014]. How cPKC mediates FADD-induced Notch signaling remains unknown.

Here, we show that FADD is also involved in myoblast differentiation and skeletal muscle regeneration. A constitutively phosphoryl-mimicking mutation of FADD (FADD-D) promotes the phosphorylation of PKCa, which stabilizes Notch-1, leading to the inhibition of $\beta$-catenin accumulation and compromised regeneration of muscles. Inhibition of Notch or restoration of Wnt signaling rescues regeneration efficiency in FADD-D muscles. Furthermore, PKCa interacts with full-length Notch-1 as determined by the phosphorylation state of PKCa to suppress ligand-independent Notch-1 degradation. These data collectively suggest a novel mechanism for Notch signaling regulation during muscle regeneration.

\section{Materials and Methods}

\section{Cell Culture, Plasmids and Reagents}

C2C12 and 293T (ATCC, Manassas, Va., USA) cells were cultured in DMEM with $10 \%$ fetal bovine serum, penicillin and streptomycin. The full-length cDNA clone of Notch-1 was generously provided by Dr. Raphael Kopan [Schroeter et al., 1998]. Fulllength wild-type (WT) PKCa was kindly provided by Dr. Christer Larsson and cloned into the pRK5 vector as previously described [Cheng et al., 2012]. Go6976, cycloheximide (CHX), MG132, L685, 458 and BIO were purchased from Sigma-Aldrich (St. Louis, Mo., USA). Anti-PKCa, anti-myogenin and anti-total GSK3 $\beta$ were purchased from BD Biosciences (Franklin Lakes, N.J., USA). AntiFLAG and anti-HA were purchased from Sigma-Aldrich. Antimyc and anti-active $\beta$-catenin were purchased from Millipore (Billerica, Mass., USA). Anti-Notch-1 and anti-GSK3 $\beta$ (Y216) were purchased from Epitomics.

\section{Animal Care}

The FADD phosphorylation mutant knockin mice (FADD-D in $\mathrm{FADD}^{-/-}$alleles) were crossed and genotyped by PCR as described previously [Hua et al., 2003]. Adult (3-6 months old) FADD-D mice and their littermates were then used for the experiments. All the mice were housed in a specific pathogen animal facility in Nanjing Drum Tower Hospital, which is authorized by the Association for the Assessment and Accreditation of Laboratory Animal Care in Jiangsu Province. All animal care and experimental procedures were approved by the Animal Care and Use Committee of the School of Life Sciences of Nanjing University.

\section{Myoblast Isolation and Culture}

Primary myoblasts were isolated as described previously with minor modifications [Cheng et al., 2014]. Briefly, the mice were sacrificed by cervical dislocation. Skeletal muscles were cut from the hind limb and cut into pieces. Muscles were then digested with $0.2 \%$ collagenase II (Worthington, N.J., USA) in a cell culture incubator for $45 \mathrm{~min}$. The digested mixture was then filtered twice through $40-\mu \mathrm{m}$ cell strainers (BD Biosciences) and centrifuged. Myoblasts were then enriched by differential sedimentation. Primary myoblasts were counted and then cultured in 8-well chamber slides (BD Biosciences) that were coated with Matrigel (BD Biosciences) in the presence of growth medium (Ham's F-10 nutrient mixture containing $20 \%$ FBS and $1 \%$ penicillin/streptomycin with $5 \mathrm{ng} / \mathrm{ml}$ of basic fibroblast growth factor). For myoblast differentiation, the growth medium was switched to low-serum medium ( $2 \%$ horse serum in DMEM). BIO (500 nM) or Go6976 (5 $\mu \mathrm{M})$ was added to the differentiation medium $24 \mathrm{~h}$ after cell plating. To determine myoblast proliferation, Go6976 (5 $\mu \mathrm{M})$ and EdU $(10 \mu \mathrm{M})$ were added to the $\mathrm{C} 2 \mathrm{C} 12$ myoblasts and cultured for $12 \mathrm{~h}$.

\section{Muscle Injury}

The mice were anesthetized with isoflurane before the injury procedures. The skin was opened to expose the tibialis anterior (TA) muscles. The muscle injury was performed as described [Cheng et al., 2012]. Briefly, TA muscles were frozen by a 15-second application of a metallic rod precooled in liquid nitrogen. The skin was closed and the mice were kept on a warm plate until they recovered. For myoblast isolation, gastrocnemius muscles were injected with $20 \mu \mathrm{l}$ of cardiotoxin $(20 \mu \mathrm{M}$; Sigma-Aldrich) at 20 different sites. $\gamma$-Secretase inhibitor L685,458 $(1 \mu \mathrm{M}, 10 \mu \mathrm{l})$ and PKC inhibitor Go6976 $(2 \mu \mathrm{M}, 10 \mu \mathrm{l})$ were injected at the times indicated. The contralateral leg was injected with an equal volume of vehicles as the control. To determine the cross-section area of newborn myotubes, L685,458 or Go6976 was injected at 5 days postinjury and muscles were collected 7 days postinjury except where otherwise indicated.

\section{Immunoprecipitation}

Plasmids were transiently transfected into $293 \mathrm{~T}$ cells with Lipofectamine 2000 for 24 or $48 \mathrm{~h}$ following the manufacturer's instructions. Cells were then lysed in lysis buffer as previously described [Cheng et al., 2012]. Cell lysis was then immunoprecipi- 


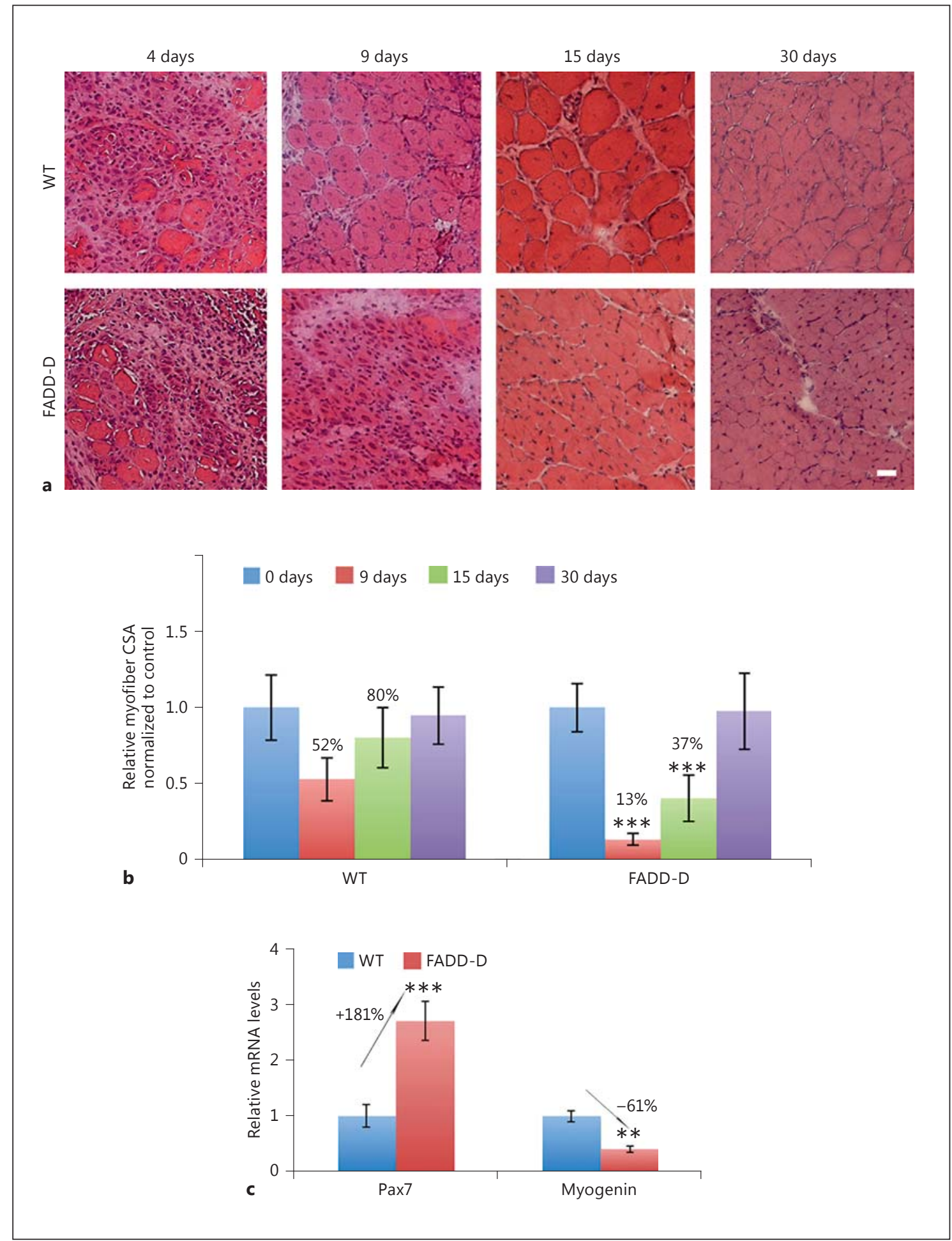

Fig. 1. FADD-D inhibits muscle regeneration. a Hematoxylin and eosin sections of injured TA muscles $(n=4)$. Scale bar $=20 \mu \mathrm{m}$. b Normalized quantification of myofiber cross-section area (CSA) from $\mathbf{a}$. The CSA from uninjured WT or FADD-D mice was set as 1 (mean $\pm \mathrm{SD}, \mathrm{n}=4$ ). c Quantitative RT-PCR analysis of primary myoblasts isolated from injured muscles at 4 days postinjury (mean $\pm \mathrm{SD}, \mathrm{n}=3$ ). 
tated with the appropriate antibody as indicated overnight. The antibody was then incubated with protein A/G-agarose (Millipore) for $2 \mathrm{~h}$ and washed 3 times with lysis buffer.

\section{Histology}

Frozen sections (10 $\mu \mathrm{m}$ thick) or cells were fixed with a freshly prepared solution of $4 \%$ paraformaldehyde in $0.1 \mathrm{M}$ phosphate buffer ( $\mathrm{pH}$ 7.2) for $10 \mathrm{~min}$. All of the images were captured by an AxioCam HR camera on an Axioplan 2 fluorescence microscope (Carl Zeiss). The fusion index was calculated as the ratio of nuclei inside the myotubes to total nuclei multiplied by 100 , and was calculated at 4 days after the start of differentiation.

\section{Quantitative RT-PCR}

Total RNA was isolated (TRIzol, Invitrogen, Carlsbad, Calif., USA) and then reverse transcribed using the SuperScript III CellsDirect cDNA Synthesis Kit (Invitrogen) to synthesize the firststrand cDNAs. Real-time RT-PCR was performed with a StepOne/ StepOnePlus ${ }^{\mathrm{TM}}$ Real-Time PCR System (Applied Biosystems) using SYBR Green PCR Master mix according to the manufacturer's instructions (Roche) with specific primers: Pax7_F: aggacgacgaggaaggagaca; Pax7_R: tcatccagacggttcccttt; Hes1_F gacaaaccaaagacggcctct; Hes1_R: ccatgataggctttgatgactttct; Myogenin_F: accaggagccccacttctatgatg; Myogenin_R: gccogccccgcctctgta; $\beta$-catenin_F: tcatgcgctccctcagat; $\beta$-catenin_R: aatggccggcttgttgc; Axin2_F: gcccgccaccaagacctacatacg; Axin2_R: cgctggtggctggtgcaaagacat; Aurora A_F: tgctgcaaacggatagggaa; Aurora A_R: acctgctccaagtttctggg; Aurora B_F: tcgctgttgtttcctctct; Aurora B_R: gatcttgagtgccacgatga; Survivin_F: catcgccaccttcaagaact; Survivin_R: agctgctcaattgactgacg; Ki67_F: agagctaacttgcgctgact; Ki67_R: actccttccaaacaggcagg.

\section{Statistical Analysis}

A minimum of 3 and a maximum of 6 replicates were prepared for all of the experiments. The data are presented as the mean and standard error of the mean (SD). Comparisons within groups were conducted using a t test with repeated measures; the $\mathrm{p}$ values indicated in the figures are: ${ }^{*} \mathrm{p}<0.05,{ }^{* *} \mathrm{p}<0.01$ and ${ }^{* * *} \mathrm{p}<0.001$.

\section{Results}

\section{FADD-D Impairs Muscle Regeneration}

Our previous studies indicated that skeletal muscles containing FADD-D exhibited a reduced myofiber size [Cheng et al., 2014]. Here, we wanted to establish whether FADD-D affected the regeneration capacity of muscles by monitoring freeze-injured TA muscle regeneration. As shown in figure 1a, muscle degeneration seemed comparable between WT and FADD-D animals at 4 days postinjury. In contrast, the size of newly formed myofibers (denoted by nuclei-centralized myofibers) in WT injured animals reached half the size of the uninjured myofibers in WT mice, whereas the newly formed myofibers in injured FADD-D animals were only 20\% the size of uninjured myofibers in FADD-D animals at 9 days postinjury (fig. 1a, b). Furthermore, the size of newly formed myofibers in WT injured animals was nearly $80 \%$ that of uninjured myofibers in WT mice at 15 days after injury. However, the size of the myofibers in injured FADD-D animals only reached $\sim 40 \%$ of that of uninjured myofibers in FADD-D mice (fig. 1a, central right and right panels, b). At 30 days postinjury, both WT and FADD-D mouse myofibers regenerated to reach their uninjured sizes (fig. 1a, b). Consistent with the delayed regeneration in FADD-D mice, myogenin, an indicator of myoblast differentiation, was significantly downregulated in regenerating myoblasts from FADD-D animals, whereas the expression of Pax7, a marker of undifferentiated myoblasts, was increased in FADD-D (fig. 1c). These data indicate that the progression of muscle regeneration is largely compromised in FADD-D animals.

\section{FADD-D Inhibits Wnt Signaling}

It has been shown that Wnt signaling promotes myofiber differentiation in physiological and aged contexts [Brack et al., 2007, 2008]. Here, we examined Wnt signaling in FADD-D muscles. Interestingly, GSK3 $\beta$ phosphorylation at Y216, which corresponds to GSK3 $\beta$ activation, was increased $\sim 2$-fold in FADD-D muscle compared with WT (fig. 2a, b). We then examined $\beta$-catenin levels in FADD-D muscle. Consistent with the increased GSK3 $\beta$ phosphorylation, protein levels of $\beta$-catenin were reduced more than $70 \%$ in FADD-D myoblasts, whereas the mRNA levels were comparable (fig. 2c). Accordingly, the transcriptional levels of Axin2, a direct transcriptional target of Wnt signaling, were also suppressed 35\% in FADD-D myoblasts from uninjured muscles (fig. 2d). Moreover, $~ 55 \%$ of $\beta$-catenin were repressed in regenerating muscles compared with WT regenerating muscle (fig. 2e), whereas its mRNA levels remained comparable (fig. 2f). These data indicate that Wnt signaling is suppressed in FADD-D muscle.

\section{Notch Inhibition Restores $\beta$-Catenin Accumulation in FADD-D Myoblasts}

Previous studies have indicated that Notch inhibits Wnt through activation of GSK3 $\beta$ during muscle regeneration [Brack et al., 2008]. Interestingly, myoblasts from regenerating FADD-D muscle indeed contained higher GSK3 $\beta$ activity (fig. $3 a$ ). We then determined whether Notch mediated GSK3 $\beta$ activation in FADD-D myoblasts. As expected, Notch inhibition by L658,458, a selective inhibitor of $\gamma$-secretase inhibitor, largely compromised the induced GSK3 $\beta$ activation in FADD-D myoblasts (fig. 3a). Consistently, $\beta$-catenin was largely restored by Notch inhibition in FADD-D cells (fig. 3a). The efficiency of Notch 


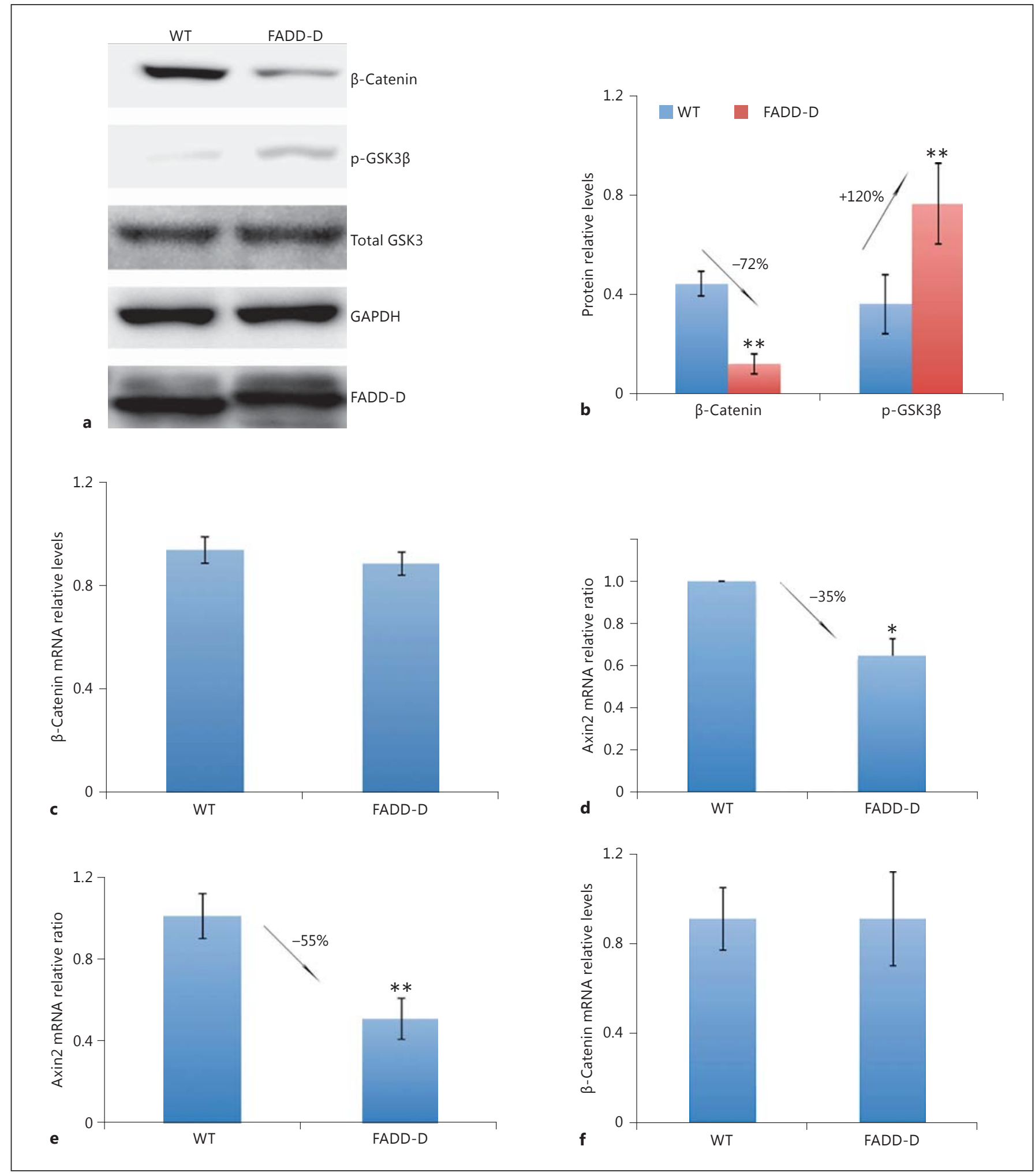

Fig. 2. FADD-D inhibits $\beta$-catenin accumulation. a Protein levels of $\beta$-catenin and phospho-GSK3 $\beta$ in primary myoblasts from uninjured muscles. Representative results were shown $(n=3)$. b Normalized quantification of $\beta$-catenin and phospho-GSK3 $\beta$ protein levels. c, $\mathbf{d}$ Quantitative RT-PCR of $\beta$-catenin and Axin2 in primary myoblasts from uninjured muscles (mean $\pm S D, n=4)$. e, f Quantitative RT-PCR of Axin 2 and $\beta$-catenin in primary myoblasts from regenerating muscles ( 4 days postinjury; mean $\pm S D, n=3$ ). 


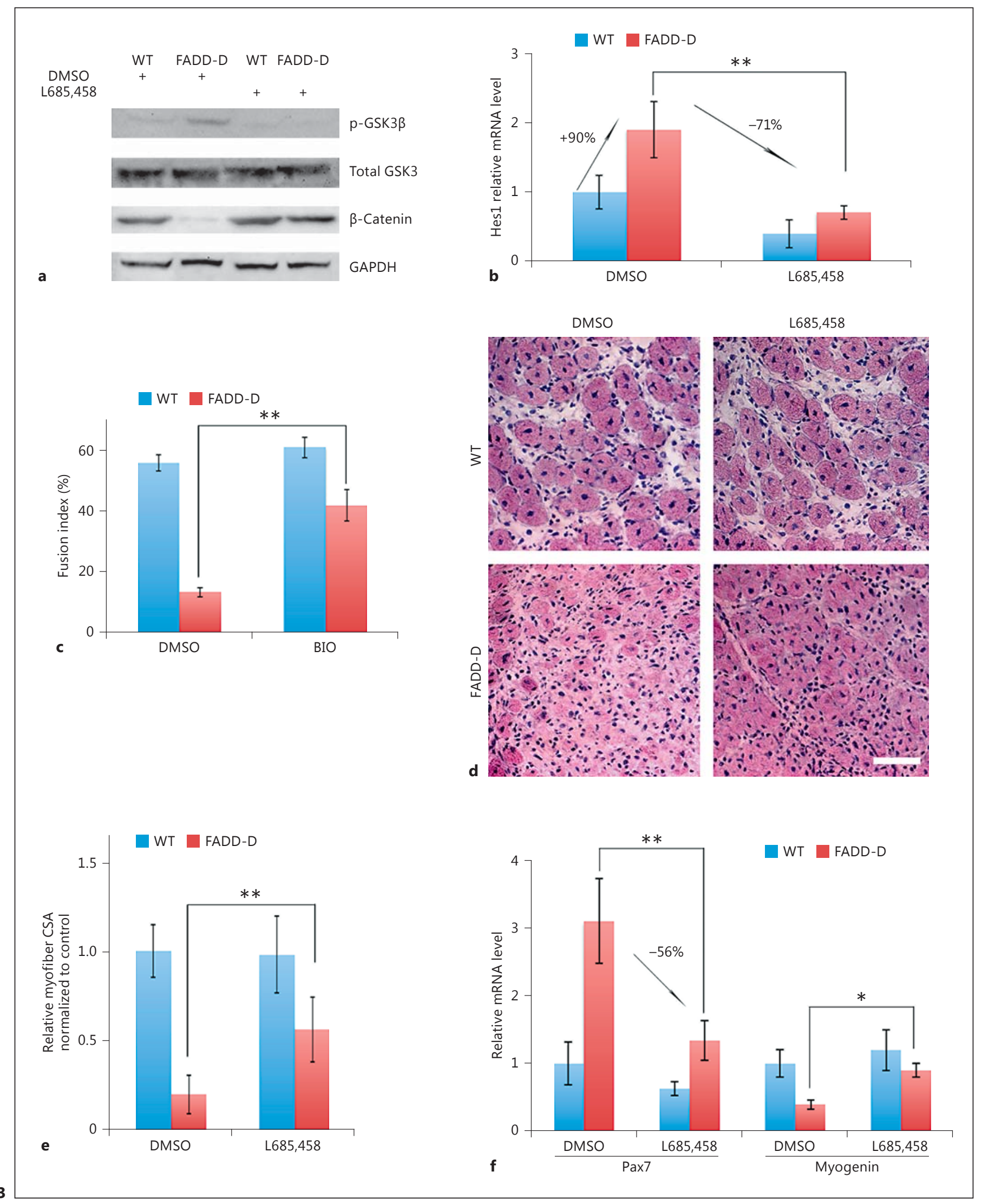

(For legend see next page.) 
inhibition was confirmed by Hes1 expression, which is a direct transcriptional target of Notch signaling (fig. 3b). Consistent with the increased Notch signaling, Hes1 was increased $\sim 2$-fold in FADD-D myoblasts compared with WT cells, while $\sim 71 \%$ of Hes 1 was repressed in myoblasts from FADD-D muscles injected with L685,458 (fig. 3b). Furthermore, a selective inhibitor of GSK3 $\beta$ (BIO) promoted the differentiation of FADD-D myoblasts in culture conditions promoting differentiation, indicating that GSK3 $\beta$ inhibits myoblast differentiation in FADD-D (fig. 3c). To examine the effects of Notch signaling on muscle regeneration in FADD-D muscle, we then determined whether Notch inhibition restored regeneration efficiency in FADD-D muscle. Indeed, Notch inhibition increased the size of newly formed myofibers in FADD-D $\sim 2.5$-fold compared with that of control FADD-D muscles injected with vehicles (fig. 3d, e). Furthermore, consistent with the restored regeneration, Pax7, the repression of which favors myoblast differentiation, was reduced $\sim 56 \%$ in FADD-D myoblasts from muscles injected with L685,458 (fig. 3f). In contrast, myogenin was increased in FADD-D myoblasts by L685,458 injection (fig. 3f). These data indicate that enhanced Notch signaling compromised Wnt signaling in FADD-D myoblasts.

PKC Promotes Notch Signaling in FADD-D Myoblasts

We have shown that FADD phosphorylation promotes PKCa activation, the inhibition of which represses the increased Notch-1 protein levels in FADD-D myogenic satellite cells [Cheng et al., 2014]. We then investigated whether PKCa also modulated Notch-1 expression in myoblasts. Compared to myoblasts isolated from the regenerating contralateral leg of FADD-D mice treated with vehicles, myoblasts exposed to a selective inhibitor of PKCa (Go6976) exhibited reduced Notch-1 levels in FADD-D (fig. 4a). The inhibition efficiency was validated by ERK phosphorylation, which is a direct effector of PKC signaling (fig. 4a). Consistently, more than $60 \%$ of

Fig. 3. Notch signaling mediates Wnt signaling in FADD-D myoblasts. a Immunoblotting of $\beta$-catenin and phospho-GSK3 $\beta$ in primary myoblasts from regenerating muscles ( 5 days postinjury) injected with L685,458 as indicated at 3.5 days postinjury. b Quantitative RT-PCR of Hes1 in samples as in a. c Quantification of cultured fused myoblasts (mean $\pm S D, n=3$ ). d Hematoxylin and eosin sections of injured TA muscles injected with L685,458; representative images are shown $(\mathrm{n}=4)$. e Normalized quantification of the myofiber cross-section area (CSA) from $\mathbf{d}$ (mean $\pm \mathrm{SD}, \mathrm{n}=$ 3). $\mathbf{f}$ Quantitative RT-PCR of Pax7 and myogenin in samples in d $($ mean $\pm S D, n=3)$. Scale bar $=20 \mu \mathrm{m}$.

FADD Regulates Notch Signaling during Muscle Regeneration
Hes1 mRNA was inhibited in FADD-D myoblasts from Go6976-treated muscles (fig. 4b). We then analyzed whether or not PKCa affected $\beta$-catenin accumulation in regenerating muscles from FADD-D mice. Notably, $\mathrm{PKC} \alpha$ inhibition restored $\beta$-catenin expression in FADD$D$ regenerating myoblasts (fig. $4 \mathrm{c}$ ). In addition, PKCa inhibition also resulted in compromised Pax7 expression in FADD-D myoblasts, while myogenin expression was restored (fig. 4d). To determine whether PKCa regulates myoblast differentiation in FADD-D cells, we monitored the differentiation of myoblasts from FADD-D muscle. As expected, inhibition of $\mathrm{PKCa}$ promoted the differentiation of myoblasts in FADD-D (fig. 4e). Thereafter, we were interested in whether the activated PKCa regulated muscle regeneration in FADD-D mice. Compared to the contralateral leg, Go6976 restored the size of regenerating myofibers efficiently in FADD-D mice (fig. $4 \mathrm{f}$ ). The newborn myofiber size was increased 4-fold by Go6976 treatment in regenerating FADD-D muscles compared to that of vehicle-injected muscle (fig. 4g). These data indicate that enhanced Notch-1 signaling in FADD-D myoblasts is mediated by PKC.

\section{PKC $\alpha$ Regulates Notch-1 Stability}

Because Notch-1 has a rapid turnover rate, we investigated whether $\mathrm{PKCa}$ could regulate the stability of Notch-1. To test this, we used CHX, which blocks mRNA translation, to monitor Notch-1 degradation. Forced expression of $\mathrm{PKCa}$ in myoblasts largely compromised Notch-1 loss induced by CHX treatment (fig. 5a). Moreover, Notch-1 protein loss induced by $\mathrm{CHX}$ was also compromised in FADD-D myoblasts compared with WT myoblasts (fig. 5b). Given the fact that Notch- 1 is a transmembrane receptor and $\mathrm{PKCa}$ also translocates to the inner cellular membrane after activation, we then went on to investigate if these two proteins interacted with each other in vivo. Interestingly, $\mathrm{PKCa}$ coimmunoprecipitated with full-length Notch-1 (fig. 5c), indicating that PKCa was able to interact with Notch-1 in vivo. Previous studies have indicated that phosphorylation events in the hydrophobic and turn motif regions of PKCa are critical for its activation and membrane translocation [Newton, 2010]. We then examined whether the phosphorylation of PKCa regulated its interaction with Notch-1. As shown in figure $5 \mathrm{~d}$ and e, constitutively phosphorylated mutations in the hydrophobic and turn motif regions of PKCa promoted its interaction with Notch-1, suggesting that the interaction between PKCa and Notch-1 is regulated by the phosphorylation status of PKCa. Furthermore, no coimmunoprecipitation was observed between the released intra- 


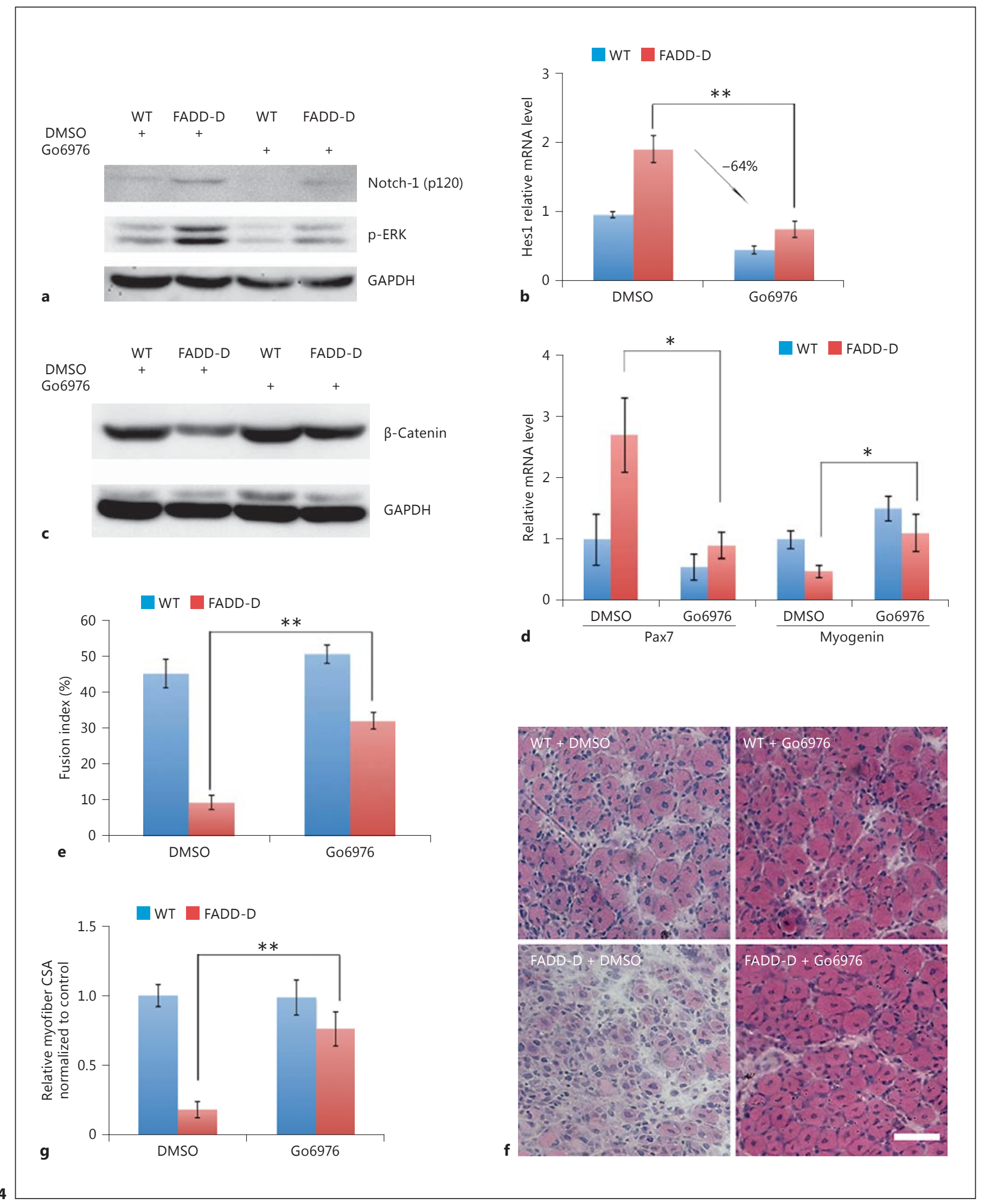

(For legend see next page.) 
cellular domain of Notch-1 (NICD) and PKCa, suggesting that PKCa only interacts with full-length Notch-1 rather than its activated form (fig. 5f). These data suggested that Notch-1 was stabilized by PKCa.

\section{PKC $\alpha$ Regulates Muscle Regeneration}

Given that PKCa and FADD phosphorylation were induced in regenerating muscles and previous studies have indicated a critical role of $\mathrm{PKCa}$ in cell proliferation [Black, 2000], we hypothesized that increased PKCa phosphorylation promotes myoblast expansion. Indeed, $\mathrm{PKCa}$ inhibition by Go6976 largely reduced nuclei-centralized myoblasts in regenerating areas, suggesting a role of $\mathrm{PKCa}$ in myoblast formation (fig. 6a, b). Consistently, cell cycle regulators including Ki67, Aurora A, survivin and Aurora B were downregulated significantly in myoblasts from Go6976-treated regenerating muscles (fig. 6c). To verify whether PKCa regulates myoblast proliferation, we examined DNA synthesis in myoblasts. PKCa inhibition suppressed DNA synthesis (denoted by EdU incorporation) and proliferation in $\mathrm{C} 2 \mathrm{C} 12$ cells and cultured primary myoblasts (fig. 6d, e), indicating that PKCa modulates myoblast proliferation. We then went on to examine whether PKCa affects differentiation. Forced PKCa expression inhibited MHC expression in myoblasts cultured in differentiation medium, suggesting that myoblast differentiation was compromised by PKCa (fig. 6f). These data suggest dual roles of PKCa in myogenic progression.

\section{Discussion}

The roles of Notch signaling in myogenic expansion have been well defined: when activated by its ligands, Notch undergoes proteolysis to release the intracellular domain, which translocates to the nucleus to activate transcriptional programs [Kopan and Ilagan, 2009]. At

Fig. 4. PKC regulates Notch in FADD-D muscles. a Regenerating gastrocnemius muscles were injected with Go6976 at 3 days postinjury for an additional 2 days. Representative results are shown $(\mathrm{n}=3)$. $\mathbf{b}$ Quantitative RT-PCR of Hes 1 in samples from $\mathbf{a}$ (mean $\pm \mathrm{SD}, \mathrm{n}=3$ ). c Protein levels of $\beta$-catenin in primary myoblasts isolated from regenerating muscles 7 days postinjury. d Quantitative RT-PCR of Pax7 and myogenin in primary myoblasts from regenerating muscle as in $\mathbf{c}($ mean $\pm S D, n=3)$. e Quantification of fused myoblasts (mean $\pm \mathrm{SD}, \mathrm{n}=3$ ). $\mathbf{f}$ Hematoxylin and eosin staining of sections from regenerating TA muscles injected with Go6976. Representative images are shown $(n=4)$. Scale bar $=20$ $\mu \mathrm{m}$. g Normalized quantification of myofiber cross-section area (CSA) from $\mathbf{f}$ (mean $\pm \mathrm{SD}, \mathrm{n}=3$ ).

FADD Regulates Notch Signaling during Muscle Regeneration the early phase of mouse muscle regeneration, one Notch ligand, Delta, is upregulated and promotes Notch-1 cleavage and Notch signaling [Conboy and Rando, 2002]. This ligand-dependent activation model has been widely accepted as the principal mechanism for the regulation of Notch signaling. In addition to this, previous studies have indicated that compromised endocytic trafficking of Notch receptor from plasma membrane leads to an increase in Notch signaling [Vaccari and Bilder, 2005; Chastagner et al., 2008]. These observations imply that the availability of Notch receptor for signaling is also involved in the regulation of Notch signaling. In the present study, our data indicate that ligand-independent Notch-1 degradation in myoblasts is controlled by PKCa. The protein level of Notch-1 has been observed to be increased in regenerating muscles compared to uninjured counterparts [Conboy et al., 2003]. Given that injury-induced muscle regeneration requires urgent Notch signaling to support immediate myoblast proliferation, it is reasonable to conclude that the availability of Notch-1 affects Notch signaling. PKCa phosphorylation is induced at the early phase of myogenic progression and also promotes increased interaction of PKCa with Notch-1. These observations suggest a mechanism for the sustainability of Notch signaling by modulating Notch-1 abundance on the plasma membrane in proliferating myoblasts. Our data show that PKC only interacts with full-length Notch-1 rather than cleaved NICD, suggesting the existence of a competence between TACE (tumor necrosis factor alpha-converting enzyme) and PKC. The recruitment of TACE might lead to disassociation between PKC and Notch-1. Further explorations are required to verify this hypothesis.

Here we also identified that PKCa inhibition resulted in myoblast proliferation repression. It has been shown that growth factors play critical roles in myoblast proliferation in regenerating muscles [Husmann et al., 1996]. As a classic intracellular effector of growth factors, PKCa may play a role in conveying these proliferative signals in vivo to facilitate myoblast proliferation. The present data showed that newborn myofiber size in FADD-D was largely restored by PKCa inhibition, which reached $80 \%$ of WT myofibers (fig. 4f, g), indicating that PKCa is a critical mediator involved in FADD-mediated muscle regeneration.

It has been shown that overexpression of PKCa induces Notch-4, instead of Notch-1, in breast cancer cells [Yun et al., 2013], which is inconsistent with our observations in myoblasts. Given that PKCa is highly expressed in breast cancer cells [Lahn et al., 2004], it is possible that the forced expressed $\mathrm{PKCa}$ is unable to interact with endogenous 

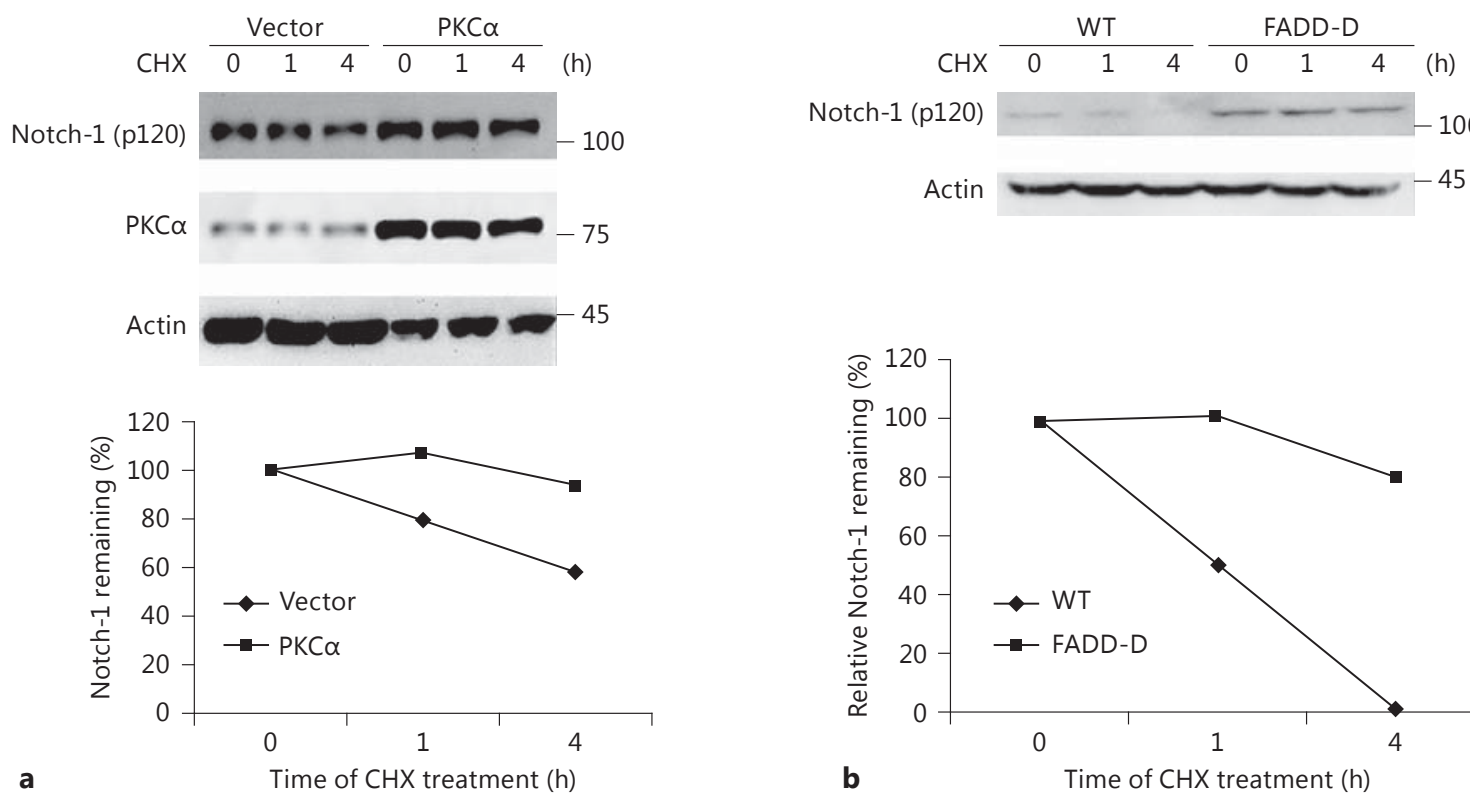

Notch-1 (p120) - - - - - - -
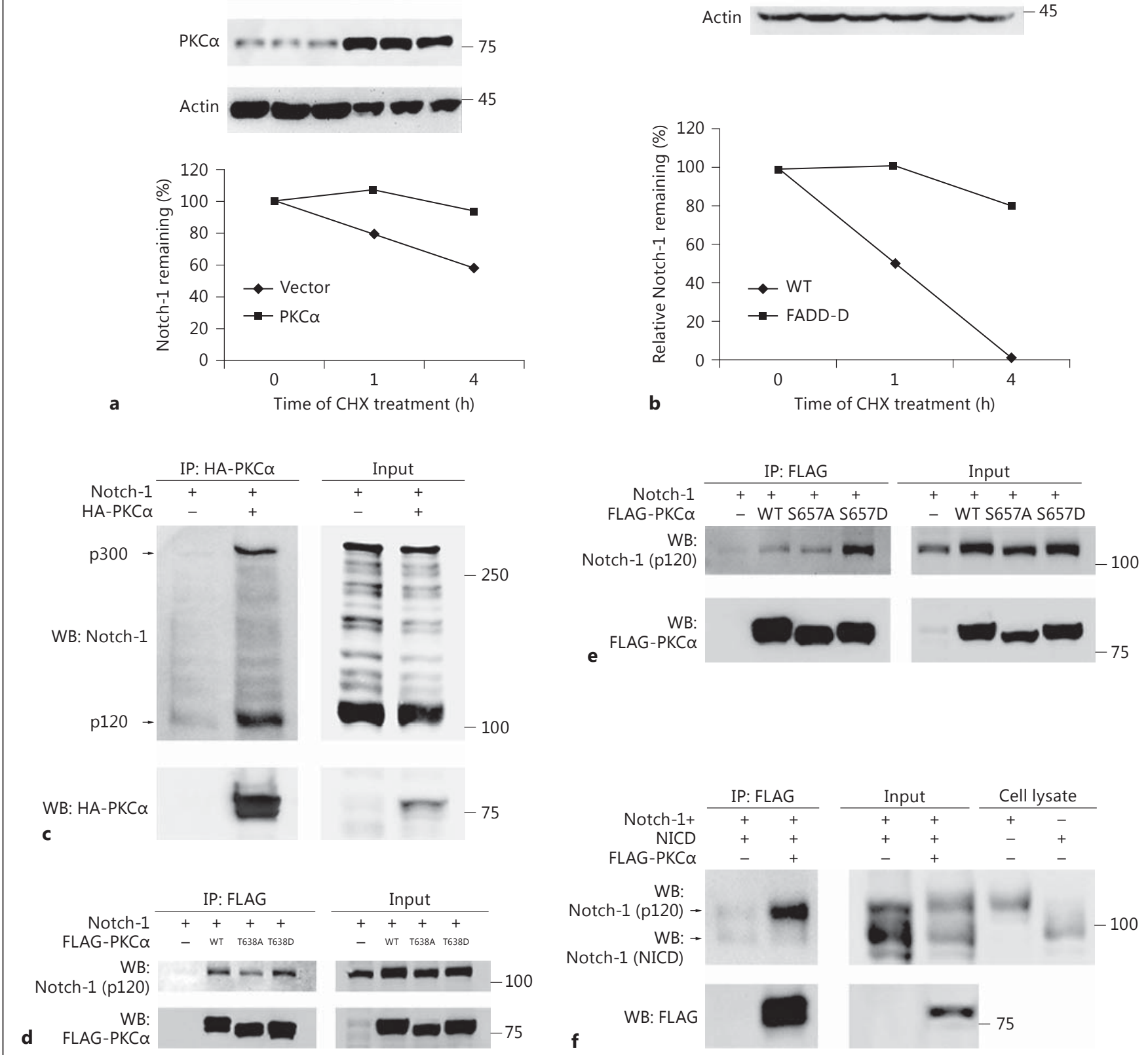

Fig. 5. PKCa regulates Notch-1 stability. a C2C12 cells expressing $\mathrm{PKC} \alpha$ or vector were treated with $\mathrm{CHX}$ (top panel). The bands were then quantified (bottom panels). b Primary myoblasts from uninjured muscles were treated with $\mathrm{CHX}$ and samples were collected at the times indicated (top panel); the bands were then quantified (bottom panels). All the experiments were performed at least three times and representative results are shown. c Coimmunopre- cipitation of PKCa with Notch-1 in 293T cells. d Coimmunoprecipitation of WT PKCa and its phosphorylation mutations in Thr638 with full-length Notch-1 in 293T cells. e Coimmunoprecipitation of WT PKCa and its phosphorylation mutations in Ser657 with Notch-1 in 293T cells. f Coimmunoprecipitation of Notch-1 and NICD with PKCa in 293T cells. The $120-\mathrm{kD}$ a portion of Notch-1 and NICD are indicated (arrowheads). 


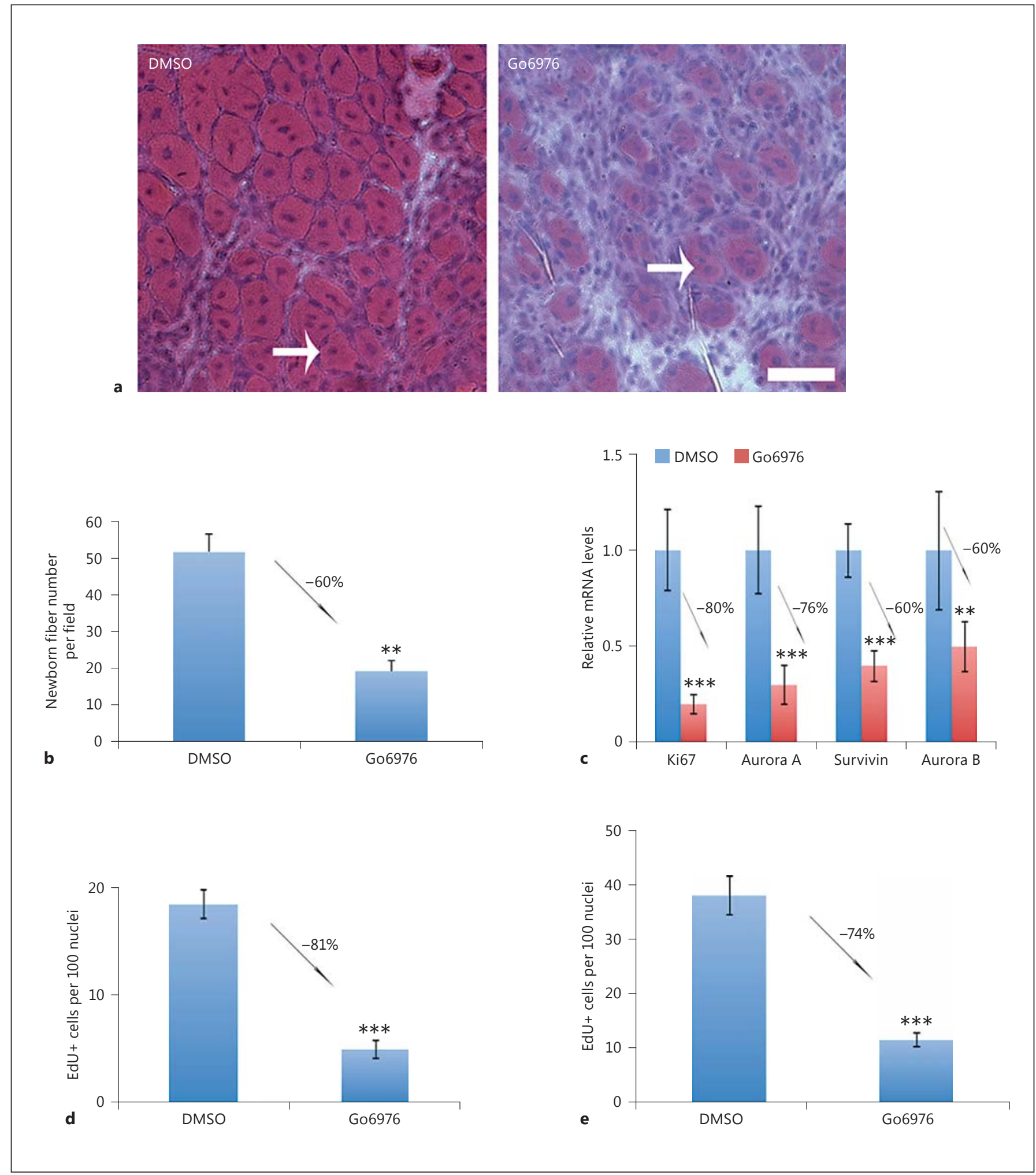

Fig. 6. PKCa regulates myogenic progression. a Regenerating muscles were injected with Go6976 at 2 days postinjury. Representative images are shown $(n=4)$. Newborn myofibers were denoted by central localized nuclei (arrows). Scale bar $=20 \mu \mathrm{m}$. b Quantification of a. c Quantitative RT-PCR of cell cycle proteins in samples from a (mean $\pm \mathrm{SD}, \mathrm{n}=3$ ). d C2C12 myoblast proliferation in the presence of Go6976. e Primary myoblast cell proliferation in the presence of Go6976 (mean \pm SD, $n=3)$. 


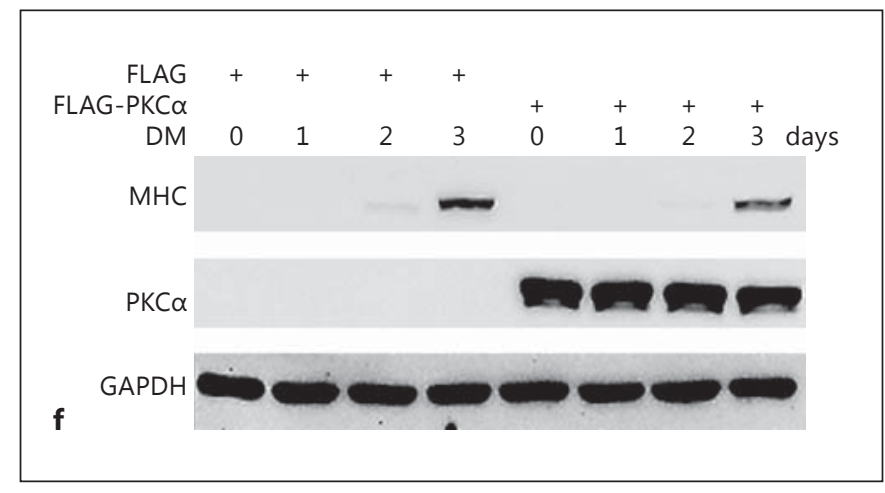

Fig. 6. PKCa regulates myogenic progression. $\mathbf{f}$ Protein levels of $\mathrm{MHC}$ in $\mathrm{PKCa}$ overexpressed $\mathrm{C} 2 \mathrm{C} 12$ cultured in differentiation medium.

Notch-1, which already binds enough PKCa in breast cancer cells. Alternatively, this discrepancy also suggests the effects of PKCa on Notch-1 depend on the cell context.

It remains unknown how Notch promotes GSK3 $\beta$ activation. Notch has been shown to inhibit AKT phosphorylation by facilitating AKT dephosphorylation [Hales et al., 2013]. AKT plays classical inhibitory roles in GSK3 $\beta$ activation [Cohen and Frame, 2001]. More importantly, AKT is required for the initiation of myoblast differentiation [Gardner et al., 2012]. These observations suggest AKT as a candidate for mediating Notch-induced GSK3 $\beta$, which needs to be determined by further studies.

\section{Acknowledgements}

The authors are grateful for grants from the National Key Basic Research Program of the Ministry of Science and Technology (2012CB967004, 2014CB744501 and 2011CB933502), the Chinese National Nature Sciences Foundation (81121062, 30425009, 30330530 and 30270291), the Jiangsu Provincial Nature Science Foundation (BE2013630, BZ2012050 and BK2011573) and the Bureau of Science and Technology of Changzhou (CM20122003, CZ20120004, CZ20130011 and CE20135013s).

\section{Disclosure Statement}

The authors declare no conflict of interests.

\section{References}

Anderson, J.E., C.M. Mitchell, J.K. McGeachie, M.D. Grounds (1995) The time course of basic fibroblast growth factor expression in crushinjured skeletal muscles of $\mathrm{SJL} / \mathrm{J}$ and $\mathrm{BALB} / \mathrm{C}$ mice. Exp Cell Res 216: 325-334.

Black, J.D. (2000) Protein kinase C-mediated regulation of the cell cycle. Front Biosci 5: D406D423.

Brack, A.S., I.M. Conboy, M.J. Conboy, J. Shen, T.A. Rando (2008) A temporal switch from notch to Wnt signaling in muscle stem cells is necessary for normal adult myogenesis. Cell Stem Cell 2: 50-59.

Chakkalakal, J.V., K.M. Jones, M.A. Basson, A.S. Brack (2012) The aged niche disrupts muscle stem cell quiescence. Nature 490: 355-360.

Chastagner, P., A. Israel, C. Brou (2008) AIP4/Itch regulates Notch receptor degradation in the absence of ligand. PLoS One 3: e2735.

Cheng, W., L. Wang, B. Yang, R. Zhang, C. Yao, L. $\mathrm{He}, \mathrm{Z}$. Liu, P. Du, K. Hammache, J. Wen, et al. (2014) Self-renewal and differentiation of muscle satellite cells is regulated by Fas-associated death domain. J Biol Chem 289: 5040-5050. Cheng, W., L. Wang, R. Zhang, P. Du, B. Yang, H.
Zhuang, B. Tang, C. Yao, M. Yu, Y. Wang, et al. (2012) Regulation of protein kinase C inactivation by Fas-associated protein with death domain. J Biol Chem 287: 26126-26135.

Cohen, P., S. Frame (2001) The renaissance of GSK3. Nat Rev Mol Cell Biol 2: 769-776.

\section{Conboy, I.M., M.J. Conboy, G.M. Smythe, T.A. Rando (2003) Notch-mediated restoration of regenerative potential to aged muscle. Science 302: 1575-1577.}

Conboy, I.M., T.A. Rando (2002) The regulation of Notch signaling controls satellite cell activation and cell fate determination in postnatal myogenesis. Dev Cell 3: 397-409.

Gardner, S., M. Anguiano, P. Rotwein (2012) Defining Akt actions in muscle differentiation. Am J Physiol Cell Physiol 303: C1292-C1300.

Hales, E.C., S.M. Orr, A. Larson Gedman, J.W. Taub, L.H. Matherly (2013) Notch1 receptor regulates AKT protein activation loop (Thr308) dephosphorylation through modulation of the PP2A phosphatase in phosphatase and tensin homolog (PTEN)-null T-cell acute lymphoblastic leukemia cells. J Biol Chem 288: 2283622848.

Hua, Z.C., S.J. Sohn, C. Kang, D. Cado, A. Winoto (2003) A function of Fas-associated death domain protein in cell cycle progression localized to a single amino acid at its $\mathrm{C}$-terminal region. Immunity 18: 513-521.

Husmann, I., L. Soulet, J. Gautron, I. Martelly, D. Barritault (1996) Growth factors in skeletal muscle regeneration. Cytokine Growth Factor Rev 7: 249-258.
Kopan, R., M.X. Ilagan (2009) The canonical Notch signaling pathway: unfolding the activation mechanism. Cell 137: 216-233.

Lahn, M., G. Kohler, K. Sundell, C. Su, S. Li, B.M Paterson, T.F. Bumol (2004) Protein kinase C alpha expression in breast and ovarian cancer. Oncology 67: 1-10.

Newton, A.C. (2010) Protein kinase C: poised to signal. Am J Physiol Endocrinol Metab 298: E395-E402.

Otto, A., C. Schmidt, G. Luke, S. Allen, P. Valasek, F. Muntoni, D. Lawrence-Watt, K. Patel (2008) Canonical Wnt signalling induces satellite-cell proliferation during adult skeletal muscle regeneration. J Cell Sci 121: 2939-2950.

-Schroeter, E.H., J.A. Kisslinger, R. Kopan (1998) Notch-1 signalling requires ligand-induced proteolytic release of intracellular domain. $\mathrm{Na}$ ture 393: 382-386.

-Vaccari, T., D. Bilder (2005) The Drosophila tumor suppressor vps25 prevents nonautonomous overproliferation by regulating notch trafficking. Dev Cell 9: 687-698.

Yin, H., F. Price, M.A. Rudnicki (2013) Satellite cells and the muscle stem cell niche. Physiol Rev 93: 23-67.

Yun, J., A. Pannuti, I. Espinoza, H. Zhu, C. Hicks, X. Zhu, M. Caskey, P. Rizzo, G. D’Souza, K. Backus, et al. (2013) Crosstalk between PKCa and Notch- 4 in endocrine-resistant breast cancer cells. Oncogenesis 2: e60. 